\title{
Utilização da Imagem Infravermelha em Reumatologia
}

\section{Infrared Imaging Use in Rheumatology}

\author{
Marcos Leal Brioschi ${ }^{(1)}$, Lin Tchia Yeng ${ }^{(2)}$, Elda Matilde Hirose Pastor ${ }^{(3)}$, Manoel Jacobsen Teixeira ${ }^{(4)}$
}

\section{RESUMO}

A imagem infravermelha (IR) é um método diagnóstico inócuo e sem radiação iônica, capaz de quantificar objetivamente por imagem as reações inflamatórias locais do sistema músculo-esquelético. Para o diagnóstico diferencial, a imagem IR deve ser analisada em conjunto com o exame clínico e outros exames complementares. A imagem IR quantitativa é útil particularmente quando aplicada na monitoração do curso da atividade inflamatória e da terapia antiinflamatória local e sistêmica.

Palavras-chave: reumatologia, imagem infravermelha, raios infravermelhos.

\section{INTRODUÇÃO}

O infravermelho é uma radiação do espectro eletromagnético com comprimentos de onda entre $0,75 \mu \mathrm{m}$ a $100 \mu \mathrm{m}$, em meio ao limite da percepção visual do vermelho e as microondas. Dependendo de sua intensidade, elas podem ser percebidas como calor pelas terminações nervosas livres, corpúsculos de Krause e de Ruffini da pele.

A imagem infravermelha (IR) é uma técnica que capta radiação na faixa do infravermelho longo $(7,5-13 \mu \mathrm{m})$ emitido pelo corpo humano, proporcionando uma imagem da distribuição térmica da superfície cutânea. A temperatura da pele, sob condições ambientais cuidadosamente controladas, é influenciada principalmente pela atividade microcirculatória desta e pela produção de calor conduzida para a superfície gerada em tecidos mais profundos.

Conhecida desde a década de 1970, na reumatologia, pelo nome de termografia ${ }^{(1-5)}$, tem recebido nova atenção nos últimos cinco anos, sendo recentemente renomeada de imagem infravermelha (IR) devido aos novos detectores

\begin{abstract}
Infrared thermography is unique objective imaging procedure for the quantitative assessment of local inflammatory reactions in parts of the locomotor system. For differential diagnosis the thermographic results should be evaluated in conjuction with clinical examination and other technical procedures. As a means of monitoring the course of the local inflammatory activity, however, quantitative infrared thermography is a useful tool in itself, particularly during the application of local and systemic anti-inflammatory therapy.
\end{abstract}

Keywords: rheumatology, infrared imaging, infrared rays.

infravermelhos ultra-sensíveis. É um método inócuo, nãoinvasivo, não-ionizante, sem contraste, capaz de mensurar a temperatura cutânea a distância com altíssima precisão em forma de imagens de alta resolução. Essa técnica, além de digitalizar as imagens, quantifica a informação em tempo real, distinguindo diferenças de temperatura menores do que $0,07^{\circ} \mathrm{C}$ em menos de 0,03 segundo $^{(6-21)}$. Recentemente, distintos métodos de imageamento infravermelho foram descritos além do estático: dinâmico com ou sem subtração de imagem, multiespectral e hiperespectral, mapeamento de textura térmica, multimodal, fusão de sensores, fusão com imagens de ressonância magnética (MRIF) e imageamento infravermelho tridimensional com e sem $\mathrm{MRIF}^{(21)}$.

A melhor compreensão dessa tecnologia está permitindo avaliar por outra perspectiva a efetividade de tratamentos reumatológicos por via local e sistêmica ${ }^{(8,22)}$. Porém, como em qualquer teste termodinâmico, o uso da imagem IR na reumatologia exige um controle rigoroso das condições ambientais da sala de exame, principalmente para reprodutibilidade dos resultados. O recinto deve ter temperatura estável e

Trabalho realizado no Centro de Dor do Hospital das Cínicas da Faculdade de Medicina da Universidade de São Paulo (HC/FMUSP). Recebido em 24/04/06. Aprovado, após revisão, em 11/11/06.

1. Professor doutor. Pós-doutorando do Centro de Dor do HC/FMUSP. Presidente da Sociedade Brasileira de Termologia

2. Professora doutora. Médica fisiatra, responsável pelo Grupo de Dor do Instituto de Ortopedia e Traumatologia do HC/FMUSP. Membro do Centro de Dor e Liga de Dor do HC/FMUSP.

3. Professora doutora. Reumatologista do HC/FMUSP. Membro da Sociedade Brasileira de Reumatologia.

4. Professor titular. Neurocirurgião. Diretor técnico da Divisão de Neurocirurgia Funcional do Instituto de Psiquiatria do HC/FMUSP. Responsável pelo Centro de Dor do HC/FMUSP. Diretor da Liga de Dor da FMUSP e da Escola de Enfermagem da USP.

Endereço para correspondência: Marcos Leal Brioschi, Rua da Paz, 195, cj. 115, CEP 80060-160, Curitiba, PR, Brasil, e-mail: infrared@infrared.med.br. 
controlada de $18^{\circ} \mathrm{C} \pm 0,5^{\circ} \mathrm{C}$, umidade de $45 \%$ e velocidade do ar $<0,2 \mathrm{~m} / \mathrm{s}$ e de preferência fluxo laminar ${ }^{(10,23)}$.

\section{ARTRITE REUMATÓIDE}

A inflamação é um dos sinais mais importantes na reumatologia, independentemente da sua patogênese. Para avaliar corretamente a atividade das doenças inflamatórias, estabelecer e julgar a estratégia terapêutica mais adequada, um dos pontos-chave é a mensuração precisa da inflamação.

A mensuração da inflamação pode ser realizada tanto por meio da quantificação subjetiva dos sintomas clássicos de dor, edema, calor, hiperemia e limitação do movimento, quanto por quantificação objetiva humoral (proteínas de fase aguda) ou de imagem (cintilografia, imagem IR).

A partir de seu início, a artrite reumatóide (AR) tem curso imprevisível, com exacerbações espontâneas e remissões a qualquer tempo. Os estágios agudos são definidos pela inflamação que antecede as alterações radiográficas ósseas e articulares. As lesões mais precoces são encontradas na membrana sinovial que envolve a articulação. A hiperemia, devido ao aumento do fluxo sangüíneo pela membrana, ocorre à medida que a renovação celular aumenta e a membrana torna-se mais espessa.

No caso da AR, vários autores criaram índices com base em parâmetros individuais como: número de articulações dolorosas, número de articulações edemaciadas, força de aperto, duração da rigidez matinal, testes funcionais, velocidade de hemossedimentação (VHS), parâmetros humorais inflamatórios, mensuração da dor (escala visual analógica de dor - VAS) e avaliação subjetiva da condição reumática pelo médico e paciente. No entanto, sua mensuração por um único parâmetro pode falhar cedo ou mais tardiamente. Foi demonstrado, pela própria imagem IR, que o grau de inflamação articular nem sempre tem correlação com a dor, especialmente em crianças $^{(24)}$. Isso não é surpresa, uma vez que a inflamação, associada com resposta imune anormal, mesmo desencadeada por infecção bacteriana, não afeta os nervos nociceptivos diretamente no primeiro momento ${ }^{(25,26)}$. Outro aspecto é que apesar de a administração de analgésicos aliviar a dor, estes não diminuem o processo inflamatório como os antiinflamatórios ${ }^{(3)}$. Assim, para maior sensibilidade e reprodutibilidade, o mais apropriado é mensurar tanto parâmetros subjetivos quanto objetivos ${ }^{(27)}$.

Devido à dificuldade em se quantificar objetivamente a atividade inflamatória na $\mathrm{AR}$, diferentes autores têm proposto associação da imagem IR juntamente com avaliação clínica subjetiva. Quanto maior a efusão articular, maior é a

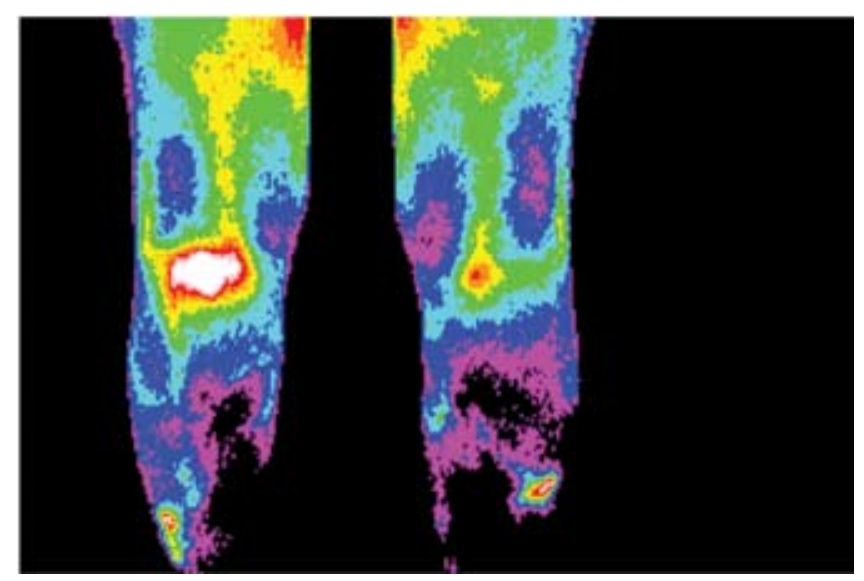

Figura 1 - Imagem hiper-radiante característica de efusão articular em razão da artrite reumatóide em joelho direito. Diferença de $3^{\circ} \mathrm{C}$ em relação ao joelho oposto. Imagem de resfriamento de apenas $-0,04^{\circ} \mathrm{C} / \mathrm{min}$ no joelho direito após estabilização térmica na sala de exames a $18^{\circ} \mathrm{C}$.

radiação infravermelha da articulação (Figura 1). No entanto, em articulações com alterações proliferativas a radiação é presente, porém um pouco menor.

$\mathrm{Na}$ fase inflamatória aguda da $\mathrm{AR}$, há padrão térmico vascular "em ferradura" ou em "U" (hiper-radiação infrapatelar) associado com patela menos hiper-radiante do que o tecido circunvizinho, devido ao derrame articular local. Já na AR crônica, o padrão térmico sobre o joelho anterior é irregular, difuso e multifocal.

Utilizando-se imagem quantitativa, por meio da mensuração de temperaturas absolutas da superfície corporal, podem-se diagnosticar e monitorar doenças reumáticas inflamatórias do sistema músculo-esquelético ${ }^{(23)}$. Para isso, o paciente deve ficar exposto semidesnudo a uma temperatura ambiente de $18^{\circ} \mathrm{C}$, e é registrada a temperatura cutânea de uma determinada área (região de interesse - ROI), por exemplo, uma articulação. As articulações inflamadas evidenciam temperaturas absolutas maiores do que as normais dentro de um tempo de exposição menor que 10 minutos com as atuais câmeras de alta resolução (imagem estática). A pele acima das articulações sadias resfria mais rapidamente e em uma extensão maior do que a pele sobre as articulações inflamadas, nas quais as temperaturas permanecem as mesmas ou diminuem muito pouco nos casos mais agudos (imagem dinâmica). Utilizando-se esses dois métodos de mensuração, é possível, então, estabelecer um diagnóstico de artrite com ajuda de normogramas-padrão com acurácia (grau de exatidão) maior do que $90 \%$ e, assim, acompanhar o curso da doença mais precisamente ${ }^{(28)}$.

A imagem dinâmica pode ser induzida pelo resfriamento da ROI com ar seco por 1 minuto seguido da avaliação 
da curva logarítmica de reaquecimento. O fluxo arterial é responsável por um rápido e primeiro reaquecimento cutâneo acompanhado de um reaquecimento mais lento, devido ao fluxo venoso patológico originário dos tecidos subcutâneos mais profundos. $\mathrm{O}$ excesso de produção de diversas substâncias vasoativas, como prostaglandinas, leucotrienos, bradicinina, histamina e especialmente óxido nítrico, pelos tecidos inflamados, associado com ativação de células $\mathrm{T}$ e outros tipos de linfócitos, é responsável por esse fluxo venoso patológico ${ }^{(18,29,30)}$.

Classicamente, há dois índices para avaliação da atividade inflamatória por imagem IR: o índice termográfico (TI), que é a análise multi-isotérmica com base na temperatura cutânea média de uma área articular pré-definida $(\mathrm{ROI})^{(3)}$, e o índice de distribuição térmica (HDI), com base em \pm 1 desvio-padrão (DP) da temperatura cutânea média dessa $\mathrm{ROI}^{(31)}$. Os valores do TI das articulações periféricas normais variam entre 0,35 e 2,30, com uma média de 1,20. O grupo de articulações em pacientes com artrite reumatóide mostrou um índice maior, com uma média de $3,96^{(4,32)}$. Estudos têm demonstrado maior sensibilidade e maior correlação do HDI em relação aos achados clínicos de gravidade da articulação afetada do que o $\mathrm{TI}^{(10,33)}$.

Devereaux et al ${ }^{(34)}$ acompanharam pacientes com AR soropositiva, com clínica clássica por 12 meses, com imagem IR de ombro, punho, joelho e tornozelo e demonstraram alta correlação entre índice de distribuição térmica (HDI) e índice articular de Ritchie, escore de Mallya, força de apreensão, rigidez matinal, VHS e VAS $(\mathrm{p}<0,001)$. Segundo os autores, o HDI é reproduzível, sensível, quantificável e não é sujeito à variação circadiana termorregulatória do paciente ou erro interobservador ${ }^{(34,35)}$. Foi demonstrada direta correlação entre diminuição do HDI e inibição da migração leucocitária em outro estudo ${ }^{(36)}$.

O exame infravermelho também é um método objetivo de avaliar resposta ao tratamento da AR com diversas drogas. Foi demonstrada marcante diminuição do HDI após injeção intra-articular de esteróides ${ }^{(6,20,37,38)}$ e somatostatina ${ }^{(15,16)} \mathrm{em}$ joelhos inflamados, mãos artríticas ${ }^{(39)}$ e pós-administração de ciclofosfamida via oral e pulsoterapia ${ }^{(36)}$, feprazona ${ }^{(40)}$, flurbiprofeno $^{(11)} \mathrm{e}$ metilprednisolona via oral em gonoartrites ${ }^{(18)}$.

Rusch et al ${ }^{(18)}$ demonstraram com o exame infravermelho que nem todos os pacientes com AR, em seu trabalho, estavam com dose suficiente de medicação antiinflamatória para remissão da doença apesar da normalização do VHS.

Diversos estudos demonstram a alta correlação entre achados clínicos, cintilografia Tc99-m e imagem IR $(\mathrm{p}<0,001)$, com alta sensibilidade nesta última ${ }^{(7,19,33,41)}$.
Warashina et $\mathrm{al}^{(19)}$ confirmaram alta correlação entre achados clínicos, radiográficos e infravermelhos após avaliarem 974 joelhos com osteoartrite $(\mathrm{p}<0,01)$.

A detecção das doenças reumáticas inflamatórias precocemente é essencial; a maior parte dos métodos convencionais para diagnóstico permite detecção apenas nas fases mais avançadas. A imagem IR é um método extremamente sensível capaz de indicar início do processo inflamatório antes da manifestação clínica. O estágio inicial da AR é caracterizado por grande proliferação da membrana sinovial e esbranquiçamento do líquido sinovial (clouding) que pode produzir imagem infravermelha detectável precocemente ${ }^{(9,14,42,43)}$. Salisbury et al avaliaram 85 pacientes com evidência clínica de sinovite em pelo menos uma articulação. De 1.362 termogramas, incluindo articulações sadias, todos os 322 com padrão alterado na distribuição térmica apresentavam sinovite clinicamente ${ }^{(31)}$. Porém, como ressalta Thumb $^{(44)}$, nada substitui um experiente reumatologista, uma vez que o diagnóstico da AR é clínico e nenhum exame isolado é diagnóstico. O primeiro estágio deve consistir da história clínica e exame físico adequado, seguido da solicitação de radiografia e exames laboratoriais específicos (segundo estágio). A imagem IR pode ser solicitada em um terceiro estágio, juntamente com exames como TC, RM, cintilografia e outros conforme o caso.

A imagem IR também tem sido utilizada na avaliação da sinovite na polimialgia reumática ${ }^{(41)}$, que é, do mesmo modo, outra doença articular relacionada à resposta imunológica $^{(45,46)}$. No tratamento da sinovite, foi descrita a complementação da imagem térmica na avaliação ultra-sonográfica, fornecendo informação da gravidade da resposta imunológica em paralelo à avaliação ultra-sonográfica da quantidade do fluido sinovial ${ }^{(47)}$.

Muitas doenças reumáticas estão associadas com disfunções do sistema nervoso neurovegetativo simpático. Foi observado que a temperatura cutânea sobre o músculo masseter é geralmente menor em indivíduos com artrite reumatóide, quando comparada com controles, apesar de estes serem livres de sintomas da articulação temporomandibular ${ }^{(48)}$. A mesma observação foi feita para articulação temporomandibular ${ }^{(49)}$, porém a explicação fisiopatológica ainda não é clara. Esses achados corroboram com observações das diferenças na distribuição térmica em diferentes áreas cutâneas de pacientes com artrite reumatóide ${ }^{(35)}$. Segundo os autores, as baixas temperaturas observadas sobre áreas musculares específicas são tão informativas quanto a avaliação das imagens hiper-radiantes das articulações inflamadas ${ }^{(2)}$. 


\section{ARTRITE REUMATÓIDE JUVENIL/ ARTRITE IDIOPÁTICA JUVENIL}

A doença de Still, uma das formas de artrite reumatóide juvenil sistêmica, é uma artrite crônica que se desenvolve na infância, podendo acometer adultos. As lesões inflamatórias são encontradas na membrana sinovial das articulações, tendões e bursas. A doença pode se apresentar inicialmente com febre elevada de origem indeterminada até dor, rigidez e edema poliarticular. O exame radiográfico é de valor limitado no diagnóstico, devido à espessa camada de cartilagem que cobre o osso jovem. Osteoporose e periostite tardia podem ser encontradas próximas às articulações inflamadas. Entretanto, um aumento térmico sobre as articulações pode ser um dos primeiros sintomas, devido à ocorrência de inflamação da membrana sinovial.

Viitanen e Laaksonen ${ }^{(50)}$, em Turku, na Finlândia, publicaram estudo interessante com 46 casos de artrite reumatóide juvenil, comparando com grupo-controle de 40 crianças da mesma idade. Como na AR do adulto, eles consideraram como patológico diferenças acima de $1^{\circ} \mathrm{C}$ das articulações. Porém, os autores notaram também dedos hiporradiantes mais freqüentemente no grupo doente do que no controle. Os achados de imagem IR se correlacionaram bem com os achados clínicos, mas, no estudo das articulações individualmente, a imagem IR foi superior ao exame clínico em 39\% dos casos. Esse número indicou diferenças térmicas patológicas antes da presença de outros sintomas clínicos ${ }^{(50)}$.

Ilowite, Walco e Pochaczevsky ${ }^{(24)}$ estudaram 18 crianças com artrite juvenil e identificaram uma correlação entre intensidade da dor e temperatura das articulações. Porém, os autores advertem que a inflamação é somente um dos fatores responsáveis pelo grau de intensidade da dor e que, em crianças com essa doença, há necessidade de compreensão de diversas outras variáveis independentes.

\section{OSTEOARTROSE}

Essa doença, embora comum, é semelhante a AR, porém ausente de sintomas dramáticos. É uma doença da cartilagem. A ordem dos eventos é indeterminada, mas as mudanças na estrutura da cartilagem levam à escamação da superfície. A face articular lesada é aberta para agressão de enzimas destrutivas da membrana sinovial. A inflamação pode ocorrer devido à irritação da membrana, causada por fissuras na cartilagem com conseqüente aumento da temperatura sobre a articulação. A extensão do aumento de temperatura geralmente é menor do que na fase aguda da artrite reumatóide ${ }^{(51)}$ e aparece como múltiplos discretos focos hiper-radiantes bem delimitados.

Em 2002, Warashina et al ${ }^{(19)}$ utilizaram índices termográficos (TI) para avaliarem, com imagem IR, 487 indivíduos normais e com osteoartrite. Eles empregaram, no estudo, índice de distribuição térmica (HDI) do joelho anterior e TI normalizado para avaliar as articulações metatarsofalangeanas (MTF) e tibiofemoral (TF). Três diferentes áreas com $3 \mathrm{~cm} \times 3 \mathrm{~cm}$ foram estudadas para o TI: $\mathrm{T}_{\mathrm{m}}$, TI da articulação MTF; $\mathrm{T}_{\mathrm{t}}$, TI da articulação TF; e $T_{p}, T I$ a $10 \mathrm{~cm}$ acima da patela. $T_{p}$ foi considerado como valor de controle. Para minimizar o efeito da variação individual da temperatura cutânea, foram calculados $T_{m}$ e $\mathrm{T}_{\mathrm{t}}\left(\Delta \mathrm{T}_{\mathrm{m}}\right.$ e $\left.\Delta \mathrm{T}_{\mathrm{t}}\right)$ subtraindo $\mathrm{T}_{\mathrm{p}}$ de $\mathrm{T}_{\mathrm{m}}$ e $\mathrm{T}_{\mathrm{t}}\left(\Delta \mathrm{T}_{\mathrm{m}}=\mathrm{T}_{\mathrm{m}}-\mathrm{T}_{\mathrm{p}}\right.$ e $\left.\Delta \mathrm{T}_{\mathrm{t}}=\mathrm{T}_{\mathrm{t}}-\mathrm{T}_{\mathrm{p}}\right)$. Os autores encontraram correlação entre HDI e edema e dor articular $(\mathrm{p}<0,01)$, porém não houve correlação com achados radiológicos. O TI normalizado correlacionou mais significativamente com os parâmetros clínicos e radiológicos (tamanho dos osteófitos) do que o HDI, sendo assim mais útil na avaliação da atividade da osteoartrite. Os autores ressaltam o potencial do TI normalizado no rastreamento da osteoartrite, porém aludem necessidade de mais estudos para confirmar sua acurácia e sensibilidade.

\section{GOTA}

A gota é uma condição causada pelo excesso de ácido úrico no sangue e nos fluidos tissulares. $\mathrm{O}$ ataque súbito produz articulações extremamente dolorosas, edematosas, sensíveis e quentes, comumente nos pés. Essa condição pode ser prontamente tratada, e a lesão desaparece tão rapidamente como apareceu. $\mathrm{O}$ efeito do tratamento na gota tem sido demonstrado por imagem IR. A redução térmica foi registrada 2 horas após administrações de indometacina, e em 24 horas a articulação estava quase assintomática ${ }^{(52,53)}$.

A gota apresenta-se como lesões hiper-radiantes nos dedos dos pés; entretanto, essas lesões não podem ser diferenciadas, simplesmente pela imagem, daquelas produzidas por artrite aguda ou fraturas. Condições locais, como infecções bacterianas ou fúngicas, paroníquia e fraturas, também produzem lesões hiper-radiantes.

\section{ARTRITE PSORIÁSICA}

Um tipo especial de doença reumática de interessante avaliação infravermelha é a artrite psoriásica, que tem sido objeto de diferentes estudos ${ }^{(54-56)}$. 
A artrite psoriásica é reconhecida como doença separada da AR. É classificada como poliartrite inflamatória associada com psoríase. Clinicamente, essa condição se assemelha com AR, mas as erupções cutâneas ( rash) não parecem estar correlacionadas com alterações artríticas ${ }^{(57)}$.

A imagem IR revela aumento da emissão térmica das placas de psoríase, devido à rápida reepitelização, de 4-7 dias, em vez dos normais 28 dias. Alguns pacientes apresentam fenômeno de Raynaud associado.

\section{ESPONDILOARTROPATIAS}

A espondilite anquilosante ocorre geralmente em homens jovens. Dor e rigidez lombar ocorrem com aumento da perda da mobilidade espinhal. Esta se espalha superiormente para a região cervical. Argawal et al ${ }^{(1)}$ encontraram hiper-radiação em 36 pacientes com espondilite anquilosante antes mesmo das alterações radiológicas. Dois pacientes deste estudo com síndrome de Reiter tinham radiografia normal, hiper-radiação infravermelha sacroilíaca e VHS aumentado. O diagnóstico radiológico das alterações inflamatórias da articulação sacroilíaca pode ser algumas vezes difícil ou dúbio, especialmente nas fases mais iniciais da doença. A imagem IR é um método extremamente sensível, capaz de identificar o começo do processo inflamatório, inclusive antes de sua manifestação clínica. É possível reconhecer um padrão térmico na sacroileíte ativa em pacientes com espondilite anquilosante e, dessa maneira, acompanhar com infravermelho a evolução da doença e seu tratamento farmacológico ${ }^{(1,5,58-60)}$. Na sacroileíte, basicamente procura-se por assimetria térmica e hiper-radiação em alguma das cinco regiões: coluna lombar, crista ilíaca, osso sacro e articulação sacroilíaca parte superior e parte inferior (Figura 2). As diferenças entre os lados direito e esquerdo podem variar de $0,5^{\circ} \mathrm{C}$ a $2^{\circ} \mathrm{C}$. A intensidade da imagem é menor nas mulheres devido à maior espessura do panículo adiposo nessa região. Muitas vezes, é acompanhada por hiporradiação na nádega no lado mais acometido (Figura 3 ).

Foi demonstrada nos pacientes com sacroileíte correlação estatisticamente significativa entre imagem IR e cintilografia (Tc99) sacroilíaca $(\mathrm{p}<0,05)^{(5,61)}$. Aliás, devido à fácil realização, pode ser repetida sem inconvenientes dos exames radiativos.

É importante, além da formação em termologia clínica e experiência em radiologia infravermelha, que o exame seja interpretado juntamente com cuidadosa avaliação clínica. Uma variedade de causas pode gerar alterações térmicas nessa região. $\mathrm{O}$ diagnóstico diferencial faz-se especialmente

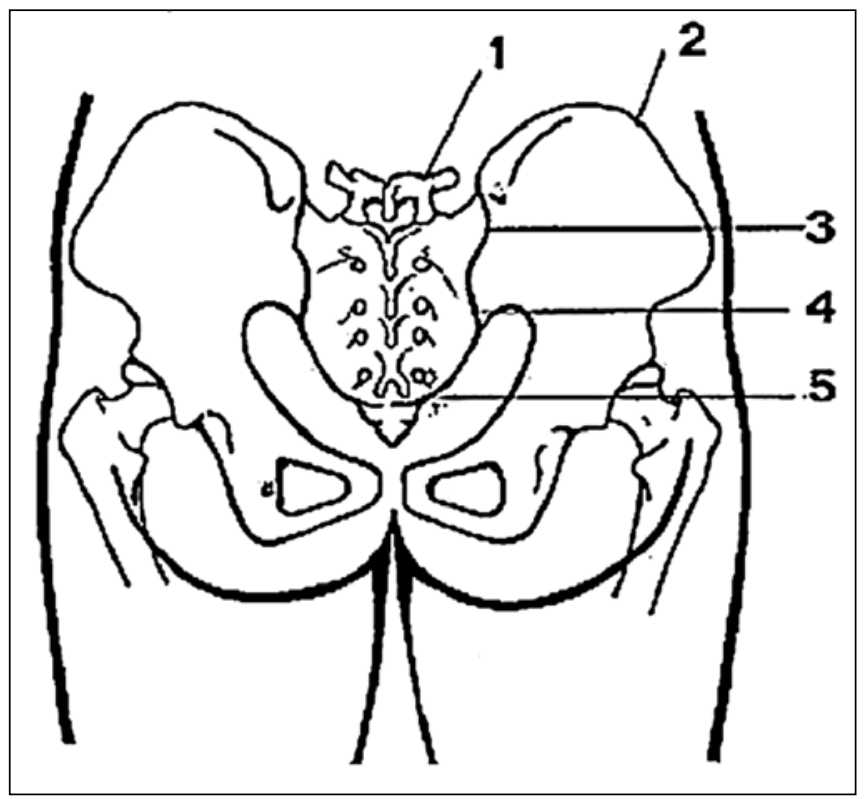

Figura 2 - Esquema das áreas avaliadas pela imagem infravermelha para diagnóstico da sacroileíte: 1) parte inferior da coluna lombar, 2) crista ilíaca, 3) parte superior da articulação sacroilíaca, 4) parte inferior da articulação sacroilíaca e 5) base do osso sacro.

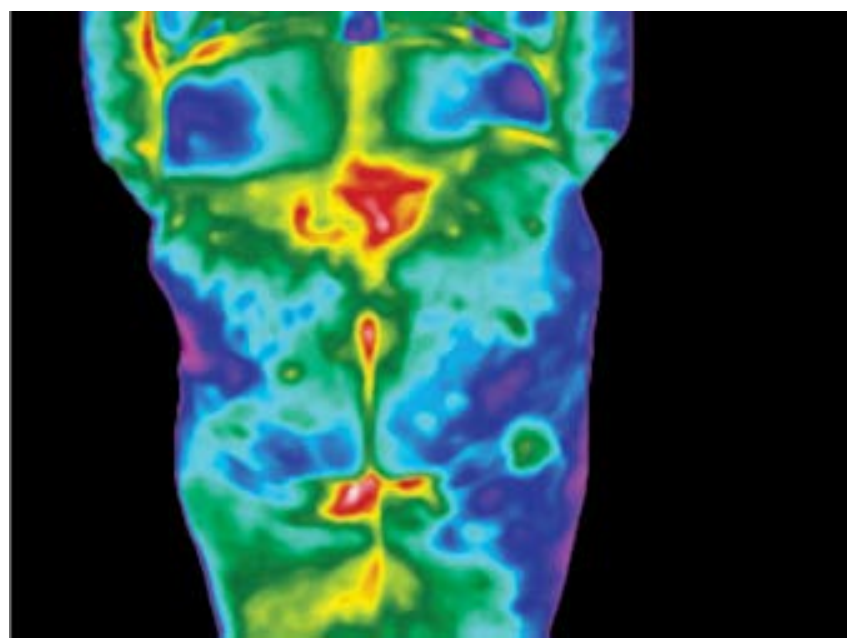

Figura 3 - Imagem hiper-radiante junto à parte superior da articulação sacroilíaca (zona 3), lado direito, característica de sacroileíte associada a hiporradiação em nádega direita.

com hérnias discais, metástases ósseas, lesões músculo-ligamentares, escoliose e condições dermatológicas.

\section{ESCLERODERMIA LOCALIZADA}

Essa doença característica é prontamente diagnosticada ao exame clínico. Na imagem IR, observa-se aumento da radiação térmica nas regiões do escleroderma, fornecendo informação do adelgaçamento do 
tecido subcutâneo que, freqüentemente, acompanha a doença. Na progressão da esclerodermia, pode ocorrer, tardiamente, fenômeno de Raynaud.

A esclerodermia localizada é doença de curso imprevisível que afeta a qualidade de vida pelo aspecto da pele e contraturas articulares. Mesmo nos três tipos principais - linear, morféia ou morféia generalizada - o infravermelho demonstra lesões hiper-radiantes ativas precocemente, auxiliando na monitoração terapêutica (Figura 4$)^{(62-64)}$.
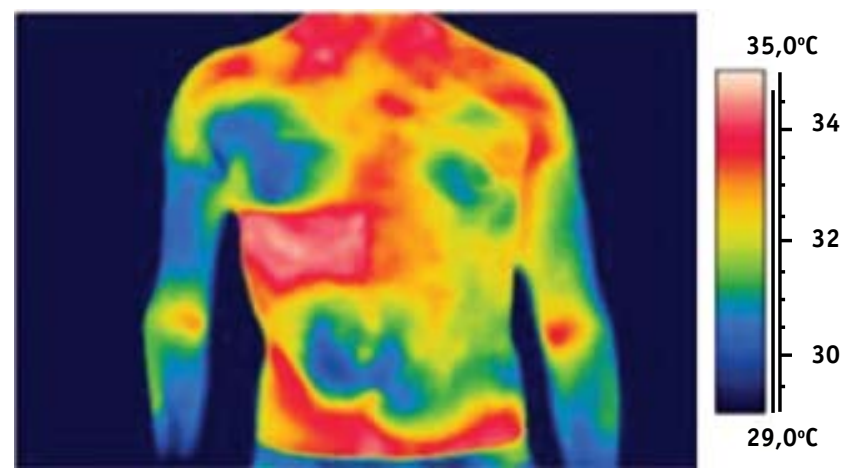

Figura 4 - Imagem hiper-radiante bem delimitada e regular em hipocôndrio direito em paciente com esclerodermia localizada.

A utilização da imagem IR na detecção de atividade na esclerodermia foi descrita pela primeira vez por Allen et al ${ }^{(65)}$. Os autores relataram caso de morféia generalizada rapidamente progressiva sob pulsoterapia de metilprednisolona. Os autores monitoraram resposta clínica e laboratorial e por imagem IR. Eles confirmaram recuperação clínica no infravermelho pelo resfriamento das lesões.

Birdi et al ${ }^{(62)}$ examinaram por imagem IR 18 lesões em 11 crianças com esclerodermia linear. Nesse estudo, três lesões extensas positivas ao exame regrediram clinicamente e ficaram negativas no infravermelho, enquanto três das 12 lesões clinicamente inalteradas permaneceram positivas no infravermelho.

Martini et al ${ }^{(63)}$ compararam imagem IR com descrição clínica da atividade da lesão em 40 crianças com esclerodermia localizada (total de 30 imagens) e encontraram sensibilidade de $92 \%$ e especificidade de $68 \%$. A especificidade foi ainda maior em lesões novas e recentes $(87,5 \%$ em lesões com menos de dois anos), com valor preditivo positivo de $87 \%$. A concordância interavaliador foi de $91 \%$ (kappa 0,82 ). Essa observação sugeriu que a imagem IR é útil na investigação da atividade da esclerodermia localizada e tem alta reprodutibilidade. Ela avalia alterações circulatórias que ocorrem nos estágios iniciais da doença, sem atrofia severa da pele e tecido subcutâneo. Portanto, pode ser utilizada na monitoração da resposta ao tratamento, como sugerida inicialmente por Allen et $a^{(65)}$.

\section{LÚPUS ERITEMATOSO CUTÂNEO}

Essa doença da pele é caracterizada por elevação difusa da temperatura cutânea além dos limites da área visível afetada. Os focos são difíceis de serem demarcados individualmente no termograma e são evidentes somente quando há grandes diferenciais térmicos, que nem sempre são paralelos ao grau de alteração cutânea visível.

\section{ERITEMA NODOSO}

Nessa forma característica de vasculite profunda, alterações cutâneas palpáveis coincidem com alterações térmicas visíveis na imagem IR. A área de hiper-radiação novamente é maior do que as alterações visíveis.

\section{DOENÇA DE PAGET (OSTEÍTE DEFORMANTE)}

Doença reumática osteolítica cujo diagnóstico precoce é muitas vezes esquecido. Os sintomas principais são dores ósseas e aumento da temperatura local. As complicações mais comuns são fraturas e insuficiência cardíaca. A imagem IR auxilia tanto no diagnóstico quanto no acompanhamento do tratamento pelo índice termográfico (TI). Avaliam-se a temperatura e o TI da tíbia anterior, região frontal do crânio, coluna vertebral e região sacroilíaca ${ }^{(66)}$.

Ring $^{(67)}$ utilizou o TI para avaliar atividade inflamatória da tíbia na doença de Paget e dosar seu tratamento com calcitonina, uma droga cara para uso prolongado e intramuscular na época. Ele observou decréscimo do TI com tratamento, assim como elevação quando este for interrompido. Não foi possível determinar a dose efetiva de calcitonina, mas antecipar manutenção da dosagem antes que ela aumentasse o TI novamente.

\section{FENÔMENO DE RAYNAUD}

As imagens térmicas podem auxiliar no diagnóstico diferencial entre o fenômeno de Raynaud (FR) primário e o secundário, por meio do teste de reaquecimento das mãos, após teste de hiper-reatividade ao frio. Há diferenças características nos padrões de distribuição térmica entre FR primário, esclerodermia (ES) e normal. Por meio das temperaturas médias das mãos, é possível plotar curva de reaquecimento indicativa do grau de disfunção circulatória (Figura 5)(68,69). 

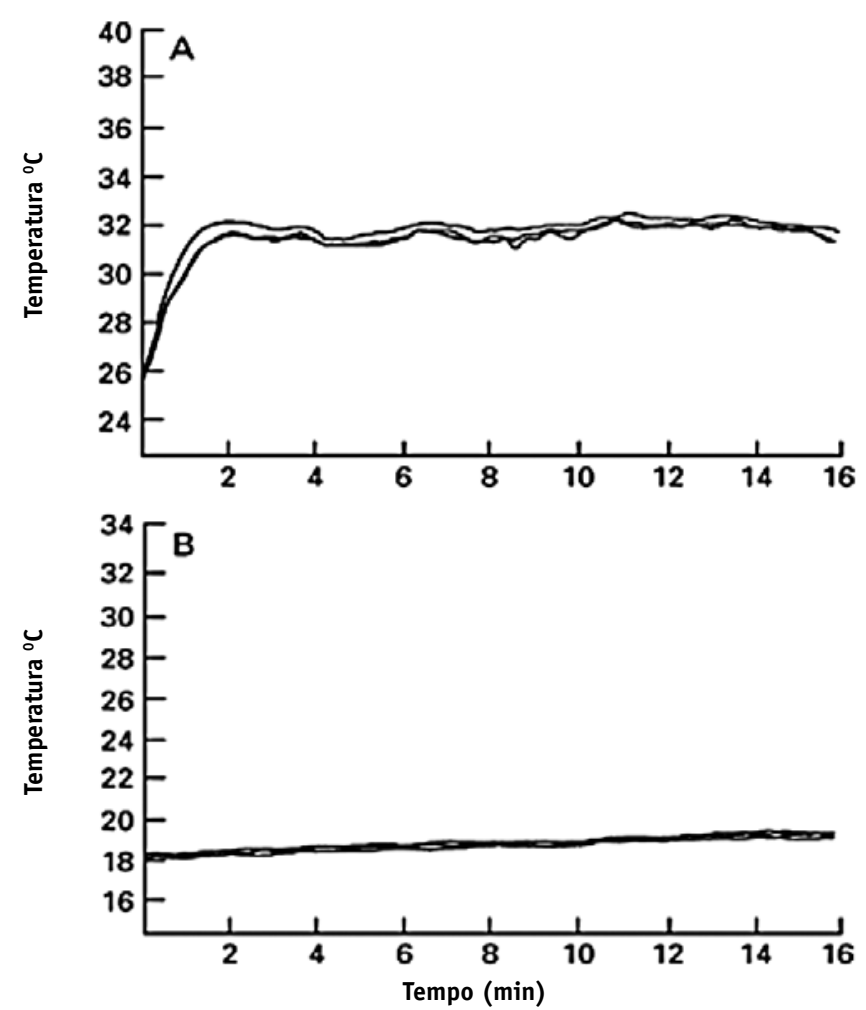

Figura 5 - Curva de recuperação (dos dedos indicador, médio e anular) de controle normal (A) e de paciente com esclerose sistêmica (B), mostrando praticamente nenhum reaquecimento (Herrick e Clark, $1998^{(69)}$ ).

O teste de hiper-reatividade ao frio (cold stress test) é realizado em sala com temperatura de $30^{\circ} \mathrm{C}$ onde o paciente mergulha ambas as mãos, protegidas em luva cirúrgica, em água a $15^{\circ} \mathrm{C}$, por 1 minuto, e observa-se curva de reaquecimento nos próximos 15 minutos. Um resfriamento maior de $1{ }^{\circ} \mathrm{C}$ dos dígitos em relação ao dorso da mão é estatisticamente significativo $(\mathrm{p}<0,005)$ para indicar doença de tecido conectivo. Sendo assim, é possível diferenciar esclerose sistêmica do FR primário por meio da imagem $\mathrm{IR}^{(70-73)}$.

Foerster et $\mathrm{al}^{(73)}$ avaliaram 139 pacientes com FR, primário e secundário, com imagem IR dos dígitos após teste de hiper-reatividade ao frio por meio de um valor "tau" de tempo de reaquecimento. Os autores encontraram valor de $8,08 \pm 3,65$ min vs $3,23 \pm 1,65$ min em controles normais, com especificidade de $94,6 \%$ e valor preditivo de $95,3 \%$.

Parece haver correlação de certos tipos de FR com deficiência na produção de óxido nítrico. A recuperação retardada, após estímulo frio, presente no FR pode ser revertida após administração oral de L-arginina ${ }^{(74-75)}$.

Foi demonstrado que o exame de imagem IR é mais específico do que o teste de Nielsen - monitoração da pressão sistólica dos dedos expostos a um ambiente frio - para avaliar $\mathrm{FR}^{(76)}$.
Diferente do laser Doppler, a imagem IR apresenta melhor correlação e resolução de imagem na avaliação do $\mathrm{FR}^{(64,77)}$, além de ser mais fácil e adequada em $\operatorname{crianças~}^{(78,79)}$. O tratamento do FR pode ser monitorado por imagem IR por meio das curvas de recuperação (reaquecimento) ${ }^{(80)}$.

A imagem IR pode avaliar ao mesmo tempo o FR e as articulações afetadas na esclerose sistêmica. Conjuntamente com a cintilografia ela demonstrou a presença de artrite em $91 \%$ e sinovite em $88 \%$ de 34 pacientes com esclerose sistêmica ${ }^{(17)}$.

\section{FIBROMIALGIA}

A imagem térmica oferece um critério objetivo no diagnóstico complementar da fibromialgia ${ }^{(81)}$, que geralmente tem sintomatologia vaga associada com componente psicossomático ${ }^{(82)}$. Esses pacientes apresentam padrão hiper-radiante não-específico correspondente às áreas musculares dolorosas clássicas, devido à hipertonia muscular local; diferente dos pontos-gatilhos miofasciais que têm imagem térmica bem regular, elipsóide e com contornos bem definidos. $\mathrm{O}$ infravermelho pode ser método diagnóstico auxiliar na documentação e acompanhamento da fibromialgia ${ }^{(83-85)}$. Também foi observado que pacientes fibromiálgicos apresentam disfunção do sistema neurovegetativo simpático. Observou-se resposta microvasoconstritiva mais fraca quando submetidos a estímulos auditivos (som alto inesperado) ou teste de hiper-reatividade ao frio do que em indivíduos normais ${ }^{(86)}$. Cerca de 20\% a 35\% dos casos apresentam FR associado ${ }^{(87)}$. Segundo Bennett et al ${ }^{(76)}$, está relacionado a distúrbios da musculatura lisa possivelmente por aumento da afinidade dos receptores alfa-2 adrenérgicos. Esses mesmos pacientes sofrem de importantes distúrbios do sono ${ }^{(82)}$ e intolerância ao frio que podem estar relacionados com disfunção da termorregulação ${ }^{(76,88)} \mathrm{e}$ explicar na imagem IR a hiper-radiação difusa que apresentam em tronco superior (em forma de "manta") em contraste com a hiporradiação de extremidades.

Também tem sido documentado o uso da imagem IR em distintas doenças reumatológicas, como epicondilites ${ }^{(89)}$, lombalgias ${ }^{(90)}$, doença de Paget ${ }^{(91)}$, entre outras.

A imagem IR é única, como método diagnóstico capaz de quantificar objetivamente por imagem de alta resolução reações inflamatórias locais do sistema músculo-esquelético. Para o diagnóstico diferencial, além da experiência do termologista, a imagem IR deve ser analisada em conjunto com exame clínico e outros exames complementares. A imagem IR quantitativa é útil particularmente quando aplicada na monitoração do curso da atividade inflamatória e da terapia antiinflamatória local e sistêmica.

Declaramos a inexistência de conflitos de interesse. 


\section{REFERÊNCIAS}

1. Agarwal A, Lloyd KN, Dovey P: Thermography of the spine and sacro-iliac joints in spondylitis. Rheumatol Phys Med 10(7):34955,1970 .

2. Huskisson EC, Berry H, Browett JP, Balme HW: Measurement of inflammation. II. Comparison of technetium clearance and thermography with standard methods in a clinical trial. Ann Rheum Dis 32: 99-102, 1973.

3. Collins AJ, Ring EF, Cosh JA, Bacon PA: Quantitation of thermography in arthritis using multi-isothermal analysis. I. The thermographic index. Ann Rheum Dis 33(2):113-5, 1974.

4. Ring EF: Thermography and rheumatic diseases. Bibl Radiol 6:97-106, 1975.

5. Sadowska-Wroblewska M, Kruszewski S, FilipowiczSosnowska A, Leo W: Use of thermography in the diagnosis of inflammatory processess of the sacroiliac joint. Reumatologia 13(3):183-91, 1975.

6. Pappalardo A, Salli L, Campisi D, Scalici G, Di Marco C, Martorana U: Telethermographic evaluation of the intra-articular administration of thymopentin in rheumatoid arthritis of the knee. Clin Ter 128(5):321-7, 1989.

7. Ignat'ev VK: The complex assessment of local inflammation in rheumatoid arthritis patients. Ter Arkh 61(12):115-7, 1989.

8. Black CM, Clark RP, Darton K, Goff MR, Norman TD, Spikes HA: A pyroelectric thermal imaging system for use in medical diagnosis. J Biomed Eng 12(4):281-6, 1990.

9. Tsvetkov AA, Kuznetsov IuA, Zotov AA: Local capillarotrophic insufficiency syndrome in patients with osteoarthrosis deformans (polarographic and thermographic data). Revmatologiia 3:1923, 1990.

10. Inoue K, Nishioka J, Kobori T, Nakatani Y, Hukuda S: The use of thermography in the assessment of the rheumatoid knee - the thermographic index and the heat distribution index. Ryumachi 30(5):356-61, 1990.

11. Young MA, Aarons L, Toon S: The pharmacokinetics of the enantiomers of flurbiprofen in patients with rheumatoid arthritis. Br J Clin Pharmacol 31(1):102-4, 1991.

12. Cervini C, Grassi W, Salaffi F: Criteria of activity of rheumatoid arthritis. Clin Ter 139(5-6):279-87, 1991.

13. Grebenik MV, Shved NI, Belozetskaia SI, Kornatskii VM: The use of thermographic and immunological indices in choosing adequate treatment for patients with rheumatoid arthritis and arthrosis deformans. Lik Sprava 3:26-9, 1992.

14. Bazhanov NN, Semenova EV, Ginzburg LI: The use of radiothermometry in patients with rheumatoid arthritis. Ter Arkh 64(2):93-6, 1992.

15. Fioravanti A, Franci A, Gelli R, Minari C, Montemerani M, Moscato P, Marcolongo R: Evaluation of the efficacy of intraarticular administration of somatostatin in rheumatoid arthritis. Clin Ter 142(5):453-7, 1993

16. Fioravanti A, Govoni M, La Montagna G, et al: Somatostatin 14 and joint inflammation: evidence for intraarticular efficacy of prolonged administration in rheumatoid arthritis. Drugs Exp Clin Res 21(3):97-103, 1995.

17. Misra R, Darton K, Jewkes RF, Black CM, Maini RN: Arthritis in scleroderma. Br J Rheumatol 34(9):831-7, 1995.
18. Rusch D, Follmann M, Boss B, Neeck G: Dynamic thermography of the knee joints in rheumatoid arthritis (RA) in the course of the first therapy of the patient with methylprednisolone. $\mathrm{Z}$ Rheumatol 59 Suppl 2:131-5, 2000.

19. Warashina $\mathrm{H}$, Hasegawa $\mathrm{Y}$, Tsuchiya $\mathrm{H}$, et al: Clinical, radiographic, and thermographic assessment of osteoarthritis in the knee joints. Ann Rheum Dis 61:852-854, 2002.

20. Balabanova RM, Fedina TP, Tsurko VV, et al: Dynamic changes in synovitis activity after intra-articular administration of xefocam in patients with rheumatoid arthritis (according to clinical and device examinations). Ter Arkh 75(5):33-5, 2003.

21. Brioschi ML, Malafaia O, Vargas JVC. Review of recent developments in thermographic applications in health care. Inframation Proceedings, Estados Unidos, v. 5, pp. 9-18, 2004.

22. Rajapakse C, Grennan DM, Jones C, Wilkinson L, Jayson M: Thermography in the assessment of peripheral joint inflammation - a re-evaluation. Rheumatol Rehabil 20(2):81-7, 1981.

23. Darton K, Black CM: The use of infra-red thermography in a rheumatology unit. Br J Rheumatol 29(4):291-2, 1990.

24. Ilowite NT, Walco GA, Pochaczevsky R: Assessment of pain in patients with juvenile rheumatoid arthritis: relation between pain intensity and degree of joint inflammation. Ann Rheum Dis 51(3):343-6, 1992.

25. Taranta A, Markowitz M: Rheumatic fever. In: McCarthy DJ, Koopman WJ, editors. Arthritis and Allied Conditions, 12th ed, Philadelphia, Lea\&Febiger, 1993.

26. Taranta A: Rheumatic fever. In: Stein JH, editor. Internal Medicine. 5th ed, St. Louis, Mosby, 1994.

27. Schattenkirchner M: Diagnostic methods for evaluation of activity in inflammatory rheumatic disease. Scand J Rheumatol Suppl 65:63-70, 1987.

28. Oblinger W, Engel JM, Franke M: Thermographic diagnosis of arthritis in peripheral joints. Z Rheumatol 44(2):77-81, 1985.

29. Kolb H, Kolb-Bachofen V: Nitric oxide: a pathogenetic factor in autoimmunity. Immunol Today 13(5):157-60, 1992.

30. Montrull HL, Brizuela NY, Demurtas SL, Spitale L, Meirovich CI: Structure and secretory activity of cultured chondrocytes from patients with osteoarthritis. Biocell 29(2):163-7, 2005.

31. Salisbury RS, Parr G, De Silva M, Hazleman BL, Page-Thomas DP: Heat distribution over normal and abnormal joints: thermal pattern and quantification. Ann Rheum Dis 42(5):494-9, 1983.

32. Collins AJ: Anti-inflammatory drug assessment by the thermographic index. Acta Thermographica 1(2):73-9, 1976.

33. De Silva M, Kyle V, Hazleman B, Salisbury R, Page Thomas P, Wraight P: Assessment of inflammation in the rheumatoid knee joint: correlation between clinical, radioisotopic, and thermographic methods. Ann Rheum Dis 45(4):277-80, 1986.

34. Devereaux MD, Parr GR, Thomas DP, Hazleman BL: Disease activity indexes in rheumatoid arthritis; a prospective, comparative study with thermography. Ann Rheum Dis 44(7):434-7, 1985

35. Teich A, Hantzschel H, Otto W, Walther H, Winiecki P: Thermologic studies in inflammatory rheumatic diseases-general presentation and personal results in rheumatoid arthritis. $\mathrm{Z}$ Gesamte Inn Med 42(22):633-8, 1987. 
36. Hall ND, Bird HA, Ring EF, Bacon PA: A combined clinical and immunological assessment of four cyclophosphamide regimes in rheumatoid arthritis. Agents Actions 9(1):97-102, 1979.

37. Thomas D, Ansell BM, Smith DS, Isaacs RJ: Knee-joint temperature measurement using a differential thermistor thermometer. Rheumatol Rehabil 19(1):8-13, 1980.

38. MacDonald AG, Land DV, Sturrock RD: Microwave thermography as a noninvasive assessment of disease activity in inflammatory arthritis. Clin Rheumatol 13(4):589-92, 1994.

39. Bird HA, Calguneri M, Leatham PA, Wright V: Measurement of temperature in the arthritic hand. Rheumatol Rehabil 19(4):20511,1980 .

40. Singer F, Gruber J, Graber J, Reinthaler M, Thumb N: The use of thermography in the evaluation of the anti-inflammatory activity of feprazone on rheumatoid arthritis. Arzneimittelforschung 32(4):427-9, 1982.

41. Kyle V, Tudor J, Wraight EP, Gresham GA, Hazleman BL: Rarity of synovitis in polymyalgia rheumatica. Ann Rheum Dis 49(3):155-7, 1990

42. Shaw RA, Kotowich S, Eysel HH, Jackson M, Thomson GT, Mantsch HH: Arthritis diagnosis based upon the near-infrared spectrum of synovial fluid. Rheumatol Int 15(4):159-65, 1995.

43. Prapavat V, Runge W, Mans J, Krause A, Beuthan J, Muller G: The development of a finger joint phantom for the optical simulation of early inflammatory rheumatic changes. Biomed Tech 42(11):319-26, 1997.

44. Thumb N: Clinical diagnostic strategy in rheumatology. Z Gesamte Inn Med 1;42(15):431-4, 1987.

45. Conn DL, Hunder GG, Duffy MO: Vasculitis and related disorders. In: Kelley WN, Harris ED, Ruddy S, Sledge CB, London WE editors. Textbook of Rheumatology. Vol 2. 4th ed, Philadelphia, Sauders, 1993, pp. 1077-1102.

46. Hunder GG: Vasculitic syndromes. In: Stein JH, editor. Internal Medicine. 5th ed, St. Louis, Mosby, 1994.

47. Van Holsbeeck M, van Holsbeeck K, Gevers G, et al: Staging and follow-up of rheumatoid arthritis of the knee. Comparison of sonography, thermography, and clinical assessment. J Ultrasound Med 7(10):561-6, 1988.

48. Tegelberg A, Kopp S: Skin surface temperature over the masseter muscle in individuals with rheumatoid arthritis. Acta Odontol Scand 46(3):151-8, 1988.

49. Tegelberg A, Kopp S: Skin surface temperature over the temporomandibular and metacarpophalangeal joints in individuals with rheumatoid arthritis. Acta Odontol Scand 45(5):329-36, 1987.

50. Viitanen SM, Laaksonen AL: Thermography in juvenile rheumatoid arthritis. Acta Rheumatol Scand 16(2):91-8, 1970.

51. Cosh JA, Ring EFJ: Thermography and rheumatology. Rheumatol Phys Med 10(7):342-8, 1970.

52. Bacon PA, Collins AJ, Ring EFJ: Quantitative thermography in the assessment of rheumatoid arthritis and gout. Acta Rheumatologica Portuguesa 2:131-4, 1974.

53. Ring EFJ, Collins AJ, Bacon PA, Cosh JA: Quantitation of thermography in arthritis using multi-isothermal analysis. II. The effect of non-steroidal anti-inflamatory therapy on the thermographic index. Ann Rheum Dis 33(4):353-6, 1974.
54. Matulis AA, Vasilenkaitis VV, Raistenskii IL, CheremnykhAlekseenko EN, Gaigalene BA: Laser therapy and laser puncture in rheumatoid arthritis, osteoarthrosis deformans and psoriatic arthropathy. Ter Arkh 55(7):92-7, 1983.

55. Loreck D, Lips H, Schulze P: Comparative clinical, $x$-ray, scintigraphic and thermographic studies in psoriasis arthropathica. Radiol Diagn 29(3):397-404, 1988.

56. McHugh NJ, Elvins DM, Ring EF: Elevated anticardiolipin antibodies in a patient with vibration-white-finger, valvular heart disease and psoriatic arthritis. Clin Rheumatol 12(1):70-3, 1993.

57. Collins AJ, Cosh JA: Temperature and biochimical studies of inflammation. Ann Rheum Dis 29:386-392, 1970.

58. Scott DG, Ring EF, Bacon PA: Problems in the assessment of disease activity in ankylosing spondylitis. Rheumatol Rehabil 20(2):74-80, 1981

59. Grennan DM, Caygill L: Infra-red thermography in the assessment of sacro-iliac inflammation. Rheumatol Rehabil 21(2):81-7, 1982.

60. Jacobsson H, Vesterskold L: The thermographic pattern of the lower back with special reference to the sacro-iliac joints in health and inflammation. Clin Rheumatol 4(4):426-32, 1985.

61. Bennett RJ, Grennan DM, Johns CW, Taylor L, Brown JD: A comparative evaluation of thermography and scintigraphy in the assessment of sacroiliitis. Int J Nucl Med Biol 11(1):42-5, 1984

62. Birdi N, Shore A, Rush P, Laxer RM, Silverman ED, Krafchik B: Childhood linear scleroderma: a possible role of thermography for evaluation. J Rheumatol 19(6):968-73, 1992.

63. Martini G, Murray KJ, Howell KJ, et al: Juvenile-onset localized scleroderma activity detection by infrared thermography. Rheumatology 41(10):1178-82, 2002.

64. Herrick AL, Hutchinson C: Vascular imaging. Best Pract Res Clin Rheumatol 18(6):957-79, 2004.

65. Allen RC, Ansell BM, Clark RP, Goff MR, Waller R, Williamson $S$ : Localized scleroderma: treatment response measured by infrared thermography. Thermology 2:550-3, 1987.

66. Crisp AJ, Smith ML, Skingle SJ, Smith M, Page Thomas DP, Hazleman BL: The localization of the bone lesions of Paget's disease by radiographs, scintigraphy and thermography: pain may be related to bone blood flow. Br J Rheumatol 28(3):266-8, 1989.

67. Ring EFJ: Thermographic evaluation of calcitonin therapy in Paget's disease of the tibia. Acta Thermographica 1(2):67-72, 1976.

68. Darton K, Black CM: Pyroelectric vidicon thermography and cold challenge quantify the severity of Raynaud's phenomenon. Br J Rheumatol 30(3):190-5, 1991.

69. Herrick AL, Clark S: Quantifying digital vascular disease in patients with primary Raynaud's phenomenon and systemic sclerosis. Ann Rheum Dis 57(2):70-8, 1998.

70. Clark S, Hollis S, Campbell F, Moore T, Jayson M, Herrick A: The "distal-dorsal difference" as a possible predictor of secondary Raynaud's phenomenon. J Rheumatol 26(5):11258, 1999

71. O'Reilly D, Taylor L, el-Hadidy K, Jayson MI: Measurement of cold challenge responses in primary Raynaud's phenomenon and Raynaud's phenomenon associated with systemic sclerosis. Ann Rheum Dis 51(11):1193-6, 1992. 
72. Schuhfried O, Vacariu G, Lang T, Korpan M, Kiener HP, Fialka-Moser V: Thermographic parameters in the diagnosis of secondary Raynaud's phenomenon. Arch Phys Med Rehabil 81(4):495-9, 2000.

73. Foerster J, Wittstock S, Fleischanderl S, et al: Infrared-monitored cold response in the assessment of Raynaud's phenomenon. Clin Exp Dermatol 31(1):6-12, 2006.

74. Agostoni A, Marasini B, Biondi ML, et al: L-arginine therapy in Raynaud's phenomenon? Int J Clin Lab Res 21(2):202-3, 1991.

75. Rembold CM, Ayers CR: Oral L-arginine can reverse digital necrosis in Raynaud's phenomenon. Mol Cell Biochem 244 (1-2):139-41, 2003.

76. Bennett RM, Clark SR, Campbell SM, et al: Symptoms of Raynaud's syndrome in patients with fibromyalgia. A study utilizing the Nielsen test, digital photoplethysmography, and measurements of platelet al.pha 2-adrenergic receptors. Arthritis Rheum 34(3):264-9, 1991.

77. Clark S, Dunn G, Moore T, Jayson M 4th, King TA, Herrick AL: Comparison of thermography and laser Doppler imaging in the assessment of Raynaud's phenomenon. Microvasc Res 66(1):73-6, 2003.

78. Quartier P: Raynaud's phenomenon in children. Arch Pediatr 11(1):74-7, 2004.

79. Biernacka-Zielinska M, Brozik H, Smolewska E, Mikinka M, Jakubowska T, Stanczyk J: Diagnostic value of thermography and endothelin concentration in serum of children with Raynaud's Syndrome. Med Wieku Rozwoj 9(2):213-22, 2005.

80. Al-Awami M, Schillinger M, Maca T, Pollanz S, Minar E: Low level laser therapy for treatment of primary and secondary Raynaud's phenomenon. Vasa 33(1):25-9, 2004.

81. Rothschild BM: Fibromyalgia: an explanation for the aches and pains of the nineties. Compr Ther 17(6):9-14, 1991.
82. Bluestein HG: Periarticular rheumatic complaints. In: Stein JH, editor. Internal Medicine. 5th ed, St. Louis, Mosby, 1994.

83. Biasi G, Fioravanti A, Franci A, Marcolongo R: The role computerized telethermography in the diagnosis of fibromyalgia syndrome. Minerva Med 85(9):451-4, 1994.

84. Sprott H, Jeschonneck M, Grohmann G, Hein G: Microcirculatory changes over the tender points in fibromyalgia patients after acupuncture therapy (measured with laser-Doppler flowmetry). Wien Klin Wochenschr 112(13):580-6, 2000.

85. Martinez-Lavin M: Fibromyalgia as a sympathetically maintained pain syndrome. Curr Pain Headache Rep 8(5):385-9, 2004.

86. Vaeroy H, Qiao ZG, Morkrid L, Forre O: Altered sympathetic nervous system response in patients with fibromyalgia (fibrositis syndrome). J Rheumatol 16(11):1460-5, 1989.

87. Dinerman H, Goldenberg DL, Felson DT: A prospective evaluation of 118 patients with the fibromyalgia syndrome: prevalence of Raynaud's phenomenon, sicca symptoms, ANA, low complement, and Ig deposition at the dermal-epidermal junction. J Rheumatol 13(2):368-73, 1986.

88. Staines DR. Is fibromyalgia an autoimmune disorder of endogenous vasoactive neuropeptides? Med Hypotheses 62(5):665-9, 2004.

89. Thomas D, Siahamis G, Marion M, Boyle C: Computerised infrared thermography and isotopic bone scanning in tennis elbow. Ann Rheum Dis 51(1):103-7, 1992.

90. Thomas D, Cullum D, Siahamis G, Langlois S: Infrared thermographic imaging, magnetic resonance imaging, CT scan and myelography in low back pain. Br J Rheumatol 29(4):26873, 1990 .

91. Wallach S: Paget's disease and fibrous dysplasia. Curr Opin Rheumatol 3(3):472-80, 1991. 